\title{
Comparative Psycholinguistic Analyses of Six Russian Poets
}

\author{
Chad C. Davidson \\ Kansas State University, Manhattan, USA
}

\begin{abstract}
The purpose of this text-based study was to determine whether the patterns of usage of Russian-written poetry reflect the poets' psychological states in the same way that a 2001 article by psychologists James Pennebaker and Shannon Stirman (P \& S) claim translated English-versions' of Russian poetry reflect the poets' psychological states. Specifically, P \& S claimed that the linguistic choices of the poet reflected whether or not he/she was suicidal. In conformity to P \& S's study, the author's study compared word-usages of matched poets. Each match consisted of one suicidal poet who matched one non-suicidal poet according to age, sex, nationality, and education. The author found a total of six matched poets (three suicidal and three non-suicidal). Poems were selected from three different periods of the poets' lives and compared accordingly. In each period, the author compared frequency of usages of seven features, six of which reflected the most relevant linguistic predictors of suicide given by Pennebaker and Stirman. Within some of the features, a number of sub-features were analyzed. Each feature and sub-feature was identified, counted, and divided against the total number of words used in the poems of the same period by the same poet. The composite list of percentages describes that poet's pattern of usage for that time period. Each finding was compared to those of their matched poet for the same time frame. Finally, the overall findings of the suicidal group were compared to the overall findings of the non-suicidal group. Interesting similarities and differences were noted along with potential for further research.
\end{abstract}

Keywords: text analysis, psycholinguistics, word count, Russian poetry

\section{Influence and Goals}

In 2001, James Pennebaker and Shannon Stirman (P \& S) published a word-based text-analysis study wherein they compared nine suicidal poets to nine non-suicidal poets. In this paper, the author discusses and compares their methods and their findings with the author's own.

The author conducted research with four primary goals in mind: (1) to see if original and pre-translated Russian poetry reveals linguistic predictors of suicide similar to predictors found in P \& S's translated English versions; (2) to see to what degree these findings imply cross-cultural characteristics of the suicidal condition; (3) to make a psychological hypothesis based on the findings, including an observation on if the suicidal condition appears lifelong or emergent; and (4) to uncover future research possibilities that could be used to fill in more knowledge gaps.

\section{Methods of Research}

P \& S first chose a group of suicidal poets whose texts they would analyze. They selected these poets by way of four criteria: (1) Only published and well-known poets for whom a sufficient amount of material could be

Chad C. Davidson, instructor at the English Language Program, Kansas State University. 
dated were included. Sufficient material was defined as having published at least two poems for each of the three periods in which P \& S split the poets' lives. The author will discuss the three periods below; (2) The poets' suicide attempts had to have been successful; (3) The poets had to have written at least two poems within one year of committing suicide, and finally, (4) Their poems must have been written in English or translated into English. Using these criteria, P \& S found one British poet, six American poets, and two Russian poets.

In order to most accurately compare the author's findings with P \& S's findings, the author used many of the same methods that they used, as well as four of the same poets. Of the four criteria that P \& S used for the suicidal poets, the author used the second criterion, "the poets' suicide attempts had to have been successful", but the author revised the other three criteria. The author's first, third, and fourth criteria were: (1) Only published and well-known poets for whom a sufficient amount of material could be dated were included. Sufficient material was defined as having published at least four poems (rather than only two), and, in general, more than 10 poems per period; (3) The poets had to have written at least four poems within one year of committing suicide; and (4) their poems had to have been written in Russian, and not translated from another language into Russian.

The author used the same suicidal Russian poets that P \& S used, which were Sergei Yesenin and Vladimir Vladimirovich Mayakovsky, but the author used only their original Russian poetry (i.e., no English translations). The author also added one more suicide poet to his list: Marina Tsvetaeva. The author added Marina Tsvetaeva, because she fit all four of the criteria listed above and because the author wanted to see if there were any differences between a female suicidal poet's word usage and the male suicidal poets' word usages.

After choosing the suicidal poets, $\mathrm{P} \& \mathrm{~S}$ found non-suicidal poets to compare them with. They chose non-suicidal poets based on how closely they matched the suicidal poets in nationality, era, education, and gender. The author used the same Russian control poets as P \& S did and followed the same criteria for finding a matched poet for Tsvetaeva. This gave the author a total of three pairs of matched poets. These matched poets were Sergei Yesenin and Boris Pasternak, Vladimir Mayakovsky and Osip Mandelshtam, and Marina Tsvetaeva and Anna Akhmatova.

P \& S selected poems from three periods: early, middle, and late. Early is defined as having been written within five years of the poet's first recorded poem. Middle is defined as having been written one to five years halfway between the earliest and final poems. And late is defined as having been written within one year of the suicidal poet's suicide and within one to eight years of the same age of their matched non-suicidal poet. The author used the same early and middle period criteria, but differed as to the late period.

$\mathrm{P} \& \mathrm{~S}$ did not compare poems written within one year of the control poets' deaths to those written within one year of the suicidal poets' deaths. For example, the late period for Sylvia Plath was a within a year of her suicide at the age of 31. Plath's control poet, Denise Levertov, wrote poetry until her death at age 74; however, her late period was considered by $\mathrm{P} \& \mathrm{~S}$ to be within eight years of when she was 31 years of age. Problematically, P \& S's "late" period comparison tells us nothing about if Levertov's poems became similar to Plath's as Levertov grew closer to death. Therefore, the author kept with P \& S's first two periods, but for the late period the author compared the poetry written during the last year of the suicidal poets with the poetry written during the last year of their non-suicidal counterparts.

\section{Comparing the Findings}

\section{P \& S's Findings}

Table 1 is a replica of the table $\mathrm{P} \& \mathrm{~S}$ gave that summarizes differences noted in their findings. The first cell 
of the table gives the semantic features followed in parentheses by example words used to count the features. The second and the third columns give findings for the Suicide Group and the findings for the Control Group, respectively. The author uses the same semantic features to analyze the original Russian poetry. Note that the numbers in the table are means in percentages; that is, feature words divided by overall words for each period.

Table 1

$P \&$ S's Table of Feature Means in Percentages

\begin{tabular}{|c|c|c|c|c|c|c|}
\hline \multirow{2}{*}{ Features } & \multicolumn{3}{|c|}{ Suicide group } & \multicolumn{3}{|c|}{ Control group } \\
\hline & Early & Middle & Late & Early & Middle & Late \\
\hline (1) I-words (e.g., me, my) & 4.00 & 3.40 & 4.00 & 2.50 & 1.60 & 2.5 \\
\hline (2) We-words (e.g., us, our) & 0.73 & 1.30 & 0.85 & 0.69 & 0.40 & 1.10 \\
\hline (3) Death words (e.g., dead, grave) & 0.52 & 0.47 & 0.69 & 0.34 & 0.43 & 0.41 \\
\hline (4) Social words (e.g., talk, share) & 1.20 & 1.10 & 1.00 & 0.89 & 1.10 & 1.30 \\
\hline (5) Positive emotion words (e.g., happy, love) & 3.30 & 3.10 & 3.90 & 2.90 & 2.90 & 2.50 \\
\hline (6) Negative emotion words (e.g., hate, anger) & 2.20 & 1.80 & 1.70 & 2.30 & 2.10 & 1.70 \\
\hline (7) Sexual words (e.g., lust, breast) & 0.60 & 0.84 & 0.47 & 0.36 & 0.36 & 0.31 \\
\hline
\end{tabular}

Note. Source: Replica of Table 1of Stirman and Pennebaker (2001, p. 518).

P \& S found that the suicidal group used more I-words (i.e., first-person singular, e.g., "myself" and "I") throughout their lives than did the control group. However, they found more We-words ("we," "us", and "our") in the early and middle periods for the suicidal group, but less in the late period. They found that Social words followed the same trend. P \& S claim that these findings support Emile Durkheim's social integration/disengagement theory of well-being which holds that suicidal individuals withdraw from social relationships and become more self-oriented (Durkheim, 1951; Schaller, 1997).

P \& S state surprise that the suicidal poets use more Positive emotion words and less Negative emotion words than the non-suicidal poets. They are also surprised by the noticeably higher number of Sexual words used by the suicidal poets throughout all phases of their careers. They do not have a hypothesis as to why this might be.

It is important to note that double entendre and metaphors are ignored in P \& S's study, and their inclusion could make a weighted difference. It is also important to point out that P \& S (and therefore the author as well) counted uses of "love" as both "Sexual words" and "Positive emotion words" features. This made for some overlap of these two features, and could explain why the suicidal group had more positive words than the control group. It might be revealing to see how different the results would be if "love" was ignored from the findings.

\section{The Author's Findings and Comparisons With P \& S's Findings}

Table 2 reveals the author's results. The author placed I-words and We-words under an overarching "Self-References" feature. I-words in this table would be more appropriately called Я-words (which include "Я," "Мной", "Меня", "моим", and the like). The author calls them I-words rather than Я-words simply to make it clear exactly what part of the author's study corresponds to what part of the P \& S's study.

It was found that the I-words increase with time for the suicidal group and decrease with time for the control group, rather than being higher or lower throughout the poets' lives. This supports Durkheim's theory, but opposes an ideology that suicidal ideations are lifelong or completely dominated by genes. It appears that the suicidal condition is an emergent process. 
Table 2

Chad C. Davidson's Table of Feature Means in Percentages

\begin{tabular}{|c|c|c|c|c|c|c|}
\hline \multirow{2}{*}{ Features } & \multicolumn{3}{|c|}{ Suicide group } & \multicolumn{3}{|c|}{ Control group } \\
\hline & Early & Middle & Late & Early & Middle & Late \\
\hline (1) Overall self-references & 3.50 & 3.72 & 4.60 & 4.07 & 3.64 & 3.09 \\
\hline Overall I-words & 2.09 & 3.10 & 3.85 & 3.65 & 2.72 & 2.52 \\
\hline Overall We-words & 1.41 & 0.62 & 0.75 & 0.42 & 0.91 & 0.57 \\
\hline (2) Death words & $\mathrm{n} / \mathrm{a}$ & 0.15 & 0.34 & $\mathrm{n} / \mathrm{a}$ & 0.34 & 0.41 \\
\hline (3) Social words & 0.34 & 0.49 & 0.48 & 0.29 & 0.52 & 0.65 \\
\hline (4) Positive emotion words & 1.09 & 1.61 & 1.64 & 1.33 & 0.99 & 0.33 \\
\hline (5) Negative emotion words & 0.40 & 0.29 & 0.44 & 1.04 & 0.46 & 0.33 \\
\hline (6) Sexual words & 0.58 & 0.84 & 1.06 & 0.83 & 0.56 & 0.41 \\
\hline (7) Negating words & 1.47 & 1.70 & 2.56 & 1.95 & 1.55 & 1.06 \\
\hline
\end{tabular}

Note. $\mathrm{n} / \mathrm{a}=$ The result was less than a hundredth of a percent.

As for We-words, unlike in P \& S's findings, in both the early and late phases, the suicidal poets use more We-words. When looking at the overall self-references results, self-reference words consistently rise through time for the suicidal poets, and lower for the control poets. It could be that the difference between poets is not an "I-centered" vs. "we-centered" mentality, as P \& S conclude, but a "self-centered" vs. "other-centered" mentality. However, this would have to be tested by future research, such as counting "Other-References" under which would be included words such as "you", "him", "her", and "they". This would be a much richer study in Russian with their greater number of pronouns. After this research has been done, we could then compare the patterns of Other-References to that of the Self-References results.

Table 3 gives a comparative synopsis of P \& S's findings to the author's own. Second in that table are Death-words. P \& S found that suicidal poets used Death words more than non-suicidal poets. The suicidal poets' talk about death picks up in the late phase, whereas the control poets' death talk slightly drops. According to psychologists Caroline Thomas and Karen Duszynski (1985), an increase in Death words may signify the presence of a death wish. Contradistinctively, the author found that Russian suicidal poets used death words less than their control poets. With all poets it picks up in the late phase, though quite a bit more so for the suicidal poets. The difference may be due to the author's using the late period of the Russian control poets the year before their deaths and P \& S's late period usually being much earlier for their control poets.

Table 3

Comparative Summary of Findings

\begin{tabular}{|l|l|}
\hline \multicolumn{1}{|c|}{ P \& S's findings } & \multicolumn{1}{c|}{ Chad C. Davidson's findings } \\
\hline Suicide group uses more I-words but less We-words. & Suicide group uses more I-words and more We-words. \\
\hline Suicide group uses more Death words. & Control group uses more Death words. \\
\hline Control group uses more Social words. & Control group uses more Social words. \\
\hline Suicide group uses more Positive emotion words. & Suicide group uses more Positive emotion words. \\
\hline Control group uses more Negative emotion words. & Control group uses more Negative emotion words. \\
\hline Suicide group uses more Sexual words. & Suicide group uses more Sexual words. \\
\hline
\end{tabular}

As mentioned above, in P \& S's study, the use of social words decreased across the suicidal group's career, while social words increased across the careers of the control poets. This trend was said to be reflected in the 
We-words findings, and that they together represented decreased interest in social relationships as poets drew closer to suicide. Similarly, the author's study revealed that social words slightly decrease in the last phase of the suicidal poets, but increase quite a bit with the control poets. Social words are higher in the suicidal poets' early phase, but higher in the control poet's middle and late phases.

As far as Positive emotion words and Negative emotion words are concerned, surprisingly, the author's study confirms P \& S's findings that suicidal poets used more positive emotion words, and less negative emotion words than the non-suicidal poets used. However, the author's results showed these patterns to an even greater degree.

As for Sexual words, the author's findings show a similar but slightly different trend to P \& S's findings. In the author's findings, Sexual words start lower in the early phase of suicidal poets, but rise substantially higher in the middle and late phases, whereas for P \& S, they were higher for suicidal poets throughout all three phases. One speculative implication from the author's findings is that as poets try to cope with feeling of intense loneliness and isolation, they react against it by creating more sexual fantasies in their poetry than ever before.

The author counted a semantic feature beyond P \& S's, which he called "Negating-words". The author counted Negating words for two reasons. The first reason was to get a very general sense of how many false positives there might be throughout the word lists, for example, at the end of Anna Akhmatova's piece, “Сколькопросьб у любимойвсегда!” (“So Many Requests for a Lover!”). In this poem, Akhmatova states, “Мнелюбвиипокоянедав" (“If I can't have love and peace”). This gives two positive emotion words: “любви" and "покоя" ("love" and "peace"). However, the two positive emotions of “любвиипокоя" are somewhat negated by the negative "недав" ("not have"). This is not revealed by a simple word count study. The author did not include the context in which he found Negating-words, however, because the author wanted to coincide with P \& S's analyses as closely as possible by not going beyond the "word". Therefore, it was only in a very speculative way that the author could view the potential number of negated feature word counts there were.

The second reason the author added Negating words was due to the concept given in the book Yes Man (2006) by Danny Wallace. That is, if saying yes more often is good for the psyche, perhaps saying "no" too often is a sign of an unhealthy or socially disengaged psyche. Interestingly, the author did find a very strong increase in Negating words as the suicidal poets neared suicide and a strong decreasing pattern of Negating word usage throughout the control poets' lives.

\section{Conclusions and Future Research Possibilities}

\section{Conclusions}

From the above combined findings, we can more accurately imply cross-cultural characteristics of the suicidal condition than we could by relying solely on the English-only word-count text-analysis done by P \& $\mathrm{S}$. We see from American, British, and Russian pre-translated poetry that the suicidal condition tends to be: (1) I/me/my[self] focused (suicidal poets used more I-words); (2) socially un-integrated/disengaged from others (control poets used more Social words while suicidal poets used more Negating words); (3) outwardly positive, perhaps to an unrealistic degree (suicidal poets used more Positive emotion words while control poets used more Negative emotion words); and (4) hyper-sensitized to the sexual (suicidal poets used more Sexual words). 


\section{Future Research Possibilities}

There is ample opportunity for future research in this field. For one, expanding the scope of the context beyond the word could prove useful. In this way, one could identify metaphors and add them to the appropriate lists, and identify which featured words the Negating words negate and add them to opposing features (e.g., "I do not have peace" = one count for the feature Negative emotion words, rather than one count feature for the Positive emotion words). In such cases, we should add something like "Construction" to the overarching feature name (e.g., "Positive Emotion words/constructions").

It could prove beneficial to count and compare all the various tense forms (past, present, and future) and maybe even the various aspectual forms and mood markers (i.e., TAM). In this way, we could see if suicidal poets are more past-centered and non-suicidal poets are more present- or future-centered and so forth.

Rather than merely counting the types of words the poets used, we could count the types of words the poets consumed and then compare their frequencies to each other, such as a text analysis of the most commonly consumed plays, songs, movies, books, letters, and discourse with friends and family. Depending on the genre, this information would, of course, be more available for modern day celebrity suicides than poets of the past. "There is nothing spontaneous, nothing natural about human desires. Our desires are artificial. We have to be taught to desire. Cinema is the ultimate pervert art; it doesn't give you what you desire; it tells you how to desire" (Fiennes, 2006). If this is the case, analyzing the types of texts consumed by modern day suicide victims, in the months (or years) before their suicide, could tell us something about what they were being told to desire.

On a more global scale, we could study how much a whole language or dialect's high frequency words (and/or constructions) tell us about that language culture's general mental state(s). Wierzbicka $(1988,1992)$ has attempted to do this with several languages. Sharifian (2003) has done so with certain Farsi key words, and calls such findings "cultural conceptualizations", i.e., the psyche of a culture as revealed in their language usage.

Once frequency of use has been identified (via corpuses comprised of native speaker discourse, if possible), then we can try to test it against the actions of the culture by asking related questions. For example, "Is the level of accessibility of a city for the disabled reflected in a higher usage of accessibility terminology in the culture's mass media?". Or more generally, "Do laws created for X follow news media usage of X-related terminology? And in turn, does mass media word usage reflect the hoi polloi usage-frequency?". This could bring about a greater understanding of one's own culture and psyche as well as higher cross-cultural awareness.

\section{References}

Durkheim, E. (1951). Suicide. New York City: Free Press.

Fiennes, S. (Director). (2006). The pervert's guide to cinema [Motion Picture]. United Kingdom, Austria, Netherlands: P Guide Ltd. \& ICA Projects.

Pennebaker, J. W. (2011). The secret life of pronouns. New York City, New York: Bloomsbury Press.

Schaller, M. (1997). The psychological consequences of fame: Three tests of the self-consciousness hypothesis. Journal of Personality and Social Psychology, 65(2), 291-309.

Sharifian, F. (2003). On cultural conceptualisations. Journal of Cognition and Culture, 3(3), 187-207.

Stirman, S. W., \& Pennebaker, J. W. (2001). Word use in the poetry of suicidal and nonsuicidal poets. Psychosomatic Medicine, 63, 517-522.

Thomas, C. B., \& Duszynski, K. R. (1985). Are words of the rorschach predictors of disease and death? The case of "whirling". Psychosomatic Medicine, 47(2), 201-211.

Wierzbicka, A. (1988). The semantics of grammar. Amsterdam: John Benjamins.

Wierzbicka, A. (1992). Semantics, culture and cognition: Universal human concepts in culture-specific configurations. New York: Oxford University Press. 